\title{
Promoting health through personal change in social networks: A German-Danish partnership
}

\author{
Petra Wihofszky* - Esslingen University of Applied Sciences, Germany \\ Annika Sternberg - University of Lübeck, Germany
}

\begin{abstract}
The project Healthy in Everyday Life is a German-Danish partnership between local health promoters and researchers from the European University of Flensburg, Germany. The objective was to promote health opportunities at the local level by qualifying citizens as health mediators, who then become active in their neighbourhoods. It was implemented in the Danish municipalities of Sønderborg and Aabenraa and the German city of Flensburg. The project processes were evaluated using participatory research methods. The project partners worked together transnationally on all stages of the project, from the recruitment of participants, to training, the development of the evaluation design and the appraisal of evaluation results. The evaluation consisted of three levels: (1) health changes on an individual level for participants; (2) impact on social environments and neighbourhoods; and (3) the transnational collaboration. This paper presents selected results. Positive developments in the health-related behaviour of the training participants were recorded. Primary networks, such as family relationships, were shown to be supportive resources. It was not possible to determine any impact on the neighbourhoods. The transnational collaboration was perceived as enriching. At the same time, there were challenges in involving the health professionals in the evaluation process, such as restricted time for joint reflection and a lack of research skills in the community practitioners. In conclusion, the project was successful in developing a health-promoting approach that received a strong response in the German and Danish municipalities involved.
\end{abstract}

Keywords: health promotion, community, social network promotion, participatory research, participation

\section{Key messages}

- The health-mediator training encouraged participants to address personal health targets and implement them in their daily lives. The participants appreciated acquiring and updating their health knowledge and reflecting on healthy lifestyles.

- A health-promoting social culture in the neighbourhoods and municipalities was stimulated by the training, small local activities and offers by the project. More time would be required to better measure the effects of the social networkpromoting approach.

- The implementation of the transnational collaboration in participatory research was complex, but successful. The participants were able to inspire and learn from each other, but this form of collaboration requires a lot of time to exchange ideas and for reflection - both at the professional level and at the level of the citizens who participated. 


\section{Background}

How can healthy lifestyles be promoted in a citizen-oriented way? This was the main question facing health professionals in the German-Danish border region and the European University of Flensburg, when they came together to develop healthpromoting approaches and to implement them through transnational collaboration. The project Healthy in Everyday Life was designed to promote citizen engagement and improve health opportunities at the neighbourhood level. Citizens with an interest in health issues were trained to become health mediators in the Danish municipalities of Sønderborg and Aabenraa and the German city of Flensburg. The project and its concomitant evaluation took place from 2013 to 2015 at these three locations. The project was financed by the European Commission in the framework of the funding line, Interreg. The goals of Interreg are to stimulate and support cross-border projects and cultural exchange in border regions. Among these are the region of South Denmark and the north of the Federal State of Schleswig-Holstein.

\section{The idea of promoting social networks}

The project Healthy in Everyday Life was oriented towards the concept of social network promotion. Social networks are defined as an interwoven mesh of social relationships (Lenz, 2000). Bruns (2013) differentiates between primary networks (natural, informal relationships), secondary networks (formalized relationships, such as memberships of clubs and associations) and tertiary networks (relationships to professionals working in the support system).

Social network promotion aims at maintaining, developing and cultivating networks, as well as shaping the conditions for the development of new networks (Trojan and Süß, 2011). With increasing individualization and pluralization of ways of life, relationships in primary networks are becoming less stable. The consequences may be social isolation and loneliness, both of which negatively impact on well-being, particularly among vulnerable groups, such as the chronically ill. These effects can be mitigated by interventions that employ network promotion.

Health promotion based on social network promotion means enhancing individuals' health resources, such as self-efficacy, by fostering social relations and inclusion in the community (Lenz, 2000; Nestmann, 2009). Individual and social resource activation affect each other reciprocally - a fact that is evident, for example, in the finding that it is easier to implement individual health targets in groups (Lenz, 2000). Network promotion can be aimed at primary networks, such as the family environment, or strengthen social cohesion in neighbourhoods. We also orient ourselves in our approach towards the model of social determinants of health (Richter and Hurrelmann, 2011; Whitehead and Dahlgren, 1991).

\section{Research findings}

The importance of social resources for health has been amply demonstrated. Social resources have a positive effect on subjective health evaluations and how people cope with disease (Hartung and Renner, 2014; Kienle et al., 2006; Knoll and Burkert, 2009). It has been shown that good neighbourhood integration enhances people's health-related well-being (Mohnen et al., 2011). Factors such as participation, satisfactory partnerships and family contacts increase the probability of engaging in healthy behaviour and using health-promoting services (Jordan and Von der Lippe, 2012; Weyers et al., 2010). Socio-economic status also affects healthy lifestyles and 
how social integration is perceived (Weyers et al., 2010). Social resources already influence changes in health behaviour at the intention phase, as shown by research into rehabilitation (Paech et al., 2014). These various research results suggest that approaches that link health-related lifestyle change and the promotion of social relations could be very promising - especially if health promotion is aimed at socially isolated people in difficult life situations (Weyers, 2008).

\section{Consequences for practice}

The central challenges for health promotion based on social network promotion are as follows: promoting the synergy of the individual and his or her social resources, strengthening primary relationships, and forging links to secondary and tertiary networks. Nestmann (2009) specifically suggests combining informal support and professional help. He coined the term 'natural helper' for socially active persons who can take a mediating role in communities and neighbourhoods. Health-promoting projects such as Community Mothers (Stadtteilmütter) in Berlin have successfully implemented such approaches (Stolzenberg et al., 2012). When implementing a network-based approach, diversity as well as differences in motivation and possibilities for participation have to be taken into account. Neighbourhoods are rarely homogeneous but, instead, show great sociodemographic diversity, differences in health-related needs and concerns, and varied forms of social interaction and relations. This applies to German and Danish municipalities and neighbourhoods (Bär, 2012; Larsen and Stock, 2011). Furthermore, we also orient ourselves towards the experiences of the project You Know What's What!, which was developed in Scandinavia and was also implemented some years ago in Flensburg (www.gesundheitliche-chancengleichheit.de/good-practice/duweisst-wo-es-lang-geht/).

\section{The transnational approach to practice}

The borderland is used by the citizens of the German and Danish communities as a joint living space, for example through work-related border commuting, the mutual use of infrastructure and public service structures, or participation in cultural events. The communities on the German and Danish sides face similar challenges, such as social and health inequities in local residential districts in which many ethnic groups live (Larsen and Stock, 2011; Wihofszky, 2013). The development of chronic illnesses is also comparable. Transnational collaboration can also contribute to creating synergies and developing new methods and approaches for mutual use. By focusing on social capital and citizen involvement, an innovative joint working basis was created to tackle and solve similar problems against the background of different societal systems.

Collaborating across the German-Danish border, the project partners developed their approach to practice. By the term 'project partner', we mean both social scientists and community practitioners working together in the project. Stimulation was provided at the primary, secondary and tertiary levels of people's social relations and networks. Training sessions for citizens with an interest in health issues in Sønderborg, Aabenraa and Flensburg were provided, and these formed the initial phase of the practical network-promoting approach. The objectives of the sessions were to enable the participants to pass on the knowledge they had gained in the training to their social environments (family, friends, colleagues and so on), and thus to contribute to an increased use of health promotion activities in their municipality, for example classes on relaxation, nutrition and exercise. Apart from individual lifestyle changes, which were initiated through setting health targets, the approach also aimed at promoting a 
culture of citizen engagement and social interaction, as well as creating links to local secondary and tertiary networks.

The training was guided by the health promotion expertise and practical experience of the Danish and German project partners, and by general theoretical concepts such as a salutogenetic understanding of health, subjective health concepts and process models for changing health-related behaviour and lifestyles (Faltermaier, 2008; Schwarzer, 2008). The exchange of expertise specific to the two countries was a plus in developing the training sessions, and made it possible to accommodate local characteristics and needs and integrate them into the training sessions.

The training was structured into three areas: (1) getting started and approaching the topic of health (this included discussions on subjective ideas about health); (2) basic, health-related knowledge (different health topics were addressed to provide a comprehensive understanding of health); and (3) personal competencies (participants focused on good and positive communication, and discussed individual health targets and their implementation). The teaching methods were diverse, and included professional input, group work, discussions of experiences and elements of personal experience. External experts were consulted on selected subjects.

There were weekly training sessions over a period of two months. Subsequently, supervision and support were offered so as to promote contacts among the training participants, to encourage the implementation of health targets and to jointly organize the participants' initial activities as health mediators in cooperation with local institutions.

\section{Evaluation design}

In parallel to the project, an evaluative research process was carried out that involved collaboration between the social scientists and community practitioners participating in the project. Participatory health research is intended to enable research to be carried out on an equal footing, and aims at the co-production of knowledge (Wright, 2013). Such a study group 'often includes engaged citizens, members of civil society (NGOs), health and social welfare professionals, health organizations, academic researchers, and policy makers' (Wright et al., 2018: 7). Participatory health research increases the usefulness of the evaluation findings. Potential disadvantages include the high demand on resources and possible divergences between science and practice (Brandes and Schaefer, 2013). Advantageous factors include the building of confidence, a shared culture of discussion between partners, mutual awareness and appreciation of each other's interests, clear roles and expectations, and the development of research skills among health professionals (Bär, 2013; Brown, 2013; Flicker et al., 2008; Hicks et al., 2012; Lantz et al., 2001). Reflexivity is seen as a key strategy, as it helps to keep the process transparent and flexible (Borg et al., 2012).

In line with the participatory research model, the Healthy in Everyday Life project partners jointly developed the whole process, ranging from recruiting training participants, developing the training sessions and the evaluation design, through to receiving feedback and discussing the findings of the evaluation. The community practitioners were also involved in gathering sociodemographic data and conducting interviews. The objectives of the process evaluation were to support the project development and implementation, to investigate the effects on personal development for the health mediators and on their social environments, and to generate recommendations for further developing the approach and transferring experience. The participation of health mediators was successively strengthened during the 
duration of the project. At the beginning, before and after interviews were conducted to record the effects of the training. Afterwards, we offered group discussions as a communicative reflection space (Bergold and Thomas, 2012) in which we exchanged ideas on the further development of the project. To this end, the evaluation design had three levels. Table 1 shows the levels with their evaluation objectives, and provides an overview of the tools and methods applied.

\section{Table 1: Overview of evaluations used at each evaluation level}

\begin{tabular}{|c|c|c|}
\hline Level & Subject of evaluation & Collection method \\
\hline Individual & $\begin{array}{l}\text { Sociodemographic profile of participants } \\
\text { Training participation } \\
\text { Implementation of training plan } \\
\text { Insights and personal experiences from the } \\
\text { training } \\
\text { Effects on daily life } \\
\text { Transfer of the training plan to a target group }\end{array}$ & $\begin{array}{l}\text { Short questionnaire } \\
\text { Registers of participants } \\
\text { Participants' observations } \\
\text { Before and after interviews } \\
\text { Structured interviews } \\
\text { Family interviews }\end{array}$ \\
\hline Group & $\begin{array}{l}\text { Role as health mediator and offers for } \\
\text { supervision }\end{array}$ & Group discussion \\
\hline Collaboration & $\begin{array}{l}\text { Quality of collaboration and process } \\
\text { assessment }\end{array}$ & Reflections of the team \\
\hline
\end{tabular}

At the individual level, the evaluation focused on the training participants, on their personal experience of the training and its effects on their daily lives, as well as on the implementation of the training plan. At the group level, the emphasis was on how participants became active as health mediators, how they addressed people in their social environments, and how they brought them and professional institutions together. The evaluation at the level of collaboration was concerned with the quality of the collaboration between the project partners.

The surveys were carried out several times during the total project duration of 22 months. With the exceptions of sociodemographic data and training participation, the surveys were conducted using qualitative methods. The participants were informed in their national language about the goals of the evaluation and about the surveys. They received initial information in the context of the training. Further information was given to the participants verbally and in writing during the survey situation. The participants' questions were addressed in person. Participation in the evaluation was voluntary. The partners who translated were informed about the confidentiality of the survey and signed a confidentiality agreement. The participants signed statements of informed consent, which could be revoked at any time. The data were digitally recorded and transcribed in the respective languages. For evaluation purposes, the Danish transcripts were translated into German. All the data, including the notes from the participant observations were analysed with MAXQDA (Kuckartz, 2014). The participants' data were anonymized. At the completion of the analysis, the data were destroyed. During the training, it was agreed that the topics and the contents discussed should remain within the framework of the training. By taking these steps, we guaranteed ethical principles to keep liabilities and risks for the participants to a minimum. As a social science project, an ethical approval application could not be submitted to the medical area of the responsible bodies in the state of SchleswigHolstein. 


\section{Collection and presentation of selected results}

The objective of the evaluation was to study the implementation of an approach in transnational collaboration involving community practitioners and researchers. The evaluation was very comprehensive. This article will present selected results that offer practically relevant insights into the implementation of the project Healthy in Everyday Life.

\section{Training participation}

Participation in the training sessions was recorded with the help of registers, kept by the local training facilitators. A total of 219 people took part in the sessions (see Table 2). Thus, the target of recruiting and training up to 50 health mediators per location was met, and was even exceeded in the Danish municipalities of Sønderborg (91 participants) and Aabenraa (78 participants).

Table 2: Number of trained participants by training groups and locations

\begin{tabular}{lcccccc}
\hline Location & Group $\mathbf{1}$ & Group 2 & Group 3 & Group 4 & Group 5 & Total \\
\hline Sønderborg & 21 & 21 & 12 & 28 & 9 & 91 \\
Aabenraa & 17 & 25 & 16 & 20 & - & 78 \\
Flensburg & 12 & 8 & 7 & 13 & 10 & 50 \\
Total & 50 & 54 & 35 & 61 & 19 & $\mathbf{2 1 9}$ \\
\hline
\end{tabular}

There were no participation restrictions with regard to age, gender, nationality and social status. However, an interest in health issues was required. Participants were mainly recruited by direct contact by key persons, but also with the help of local media, such as radio, newspapers and brochures. Participation was a voluntary offer. Thus, the participants in the training (the mediators) are not representative of their communities, even though, according to our surveys, they roughly represent them sociodemographically. In Flensburg, fewer participants could be trained than in the Danish locations, but the target of 50 health mediators was achieved. Various factors can potentially influence the number of participants, such as local supply structures, the social fabric of the local population or cultural aspects. One factor may have been different local recruitment strategies and advertising of the training, which varied from a broadly based, open offer (Flensburg) to an offer with concrete health-related behavioural changes (Sønderborg and Aabenraa). High demand for training places in the two Danish municipalities suggests that that approach was aligned with the citizens' interests, and was very well adapted to the local supply structure. In contrast to Flensburg, the training in Denmark took place in rural areas.

\section{Training participants}

A standardized short questionnaire was used to gather anonymized socio-economic data from 78.5 per cent $(n=172)$ of the training participants. The participants were predominantly female ( 86 per cent) and were 45.7 years of age on average (age range 18 to 74). The majority of participants (91.9 per cent) had the citizenship of the country they lived in (that is, Danish or German), a smaller proportion (17.5 per cent) had a background of migration. Approximately two thirds of the participants were in a relationship, and the majority (82.6 per cent) had children. Educational 
background was determined by the number of school years. Approximately half of the sample (46.2 per cent) showed medium educational attainment, 33.9 per cent high educational attainment, and 19.3 per cent low educational attainment. In comparison, Flensburg had a higher proportion of people with a background of migration and fewer participants in relationships. On average, 31 per cent of participants at all three locations received social benefits (for example, unemployment benefits, welfare allowance, housing benefits).

\section{Implementing the training plan}

The training was implemented on the basis of the training plan, developed jointly by health professionals and academic social scientists. In the second round of training, participant observations were made at all three locations to assess the implementation of the joint training plan and specific local conditions: 13 observations were made, resulting in 19 reports, which were used to extract training profiles. These observations allowed for a view of the training 'from within' (Kochinka, 2010). The results made clear that implementation was guided by the jointly developed training plan. However, it was interpreted by the training facilitators according to their skills and interests, and adapted to local conditions. In Sønderborg, the emphasis was on knowledge transfer combined with offers for physical activity. The work in Aabenraa was characterized by a consistent focus on individual health targets, and in Flensburg the training was more focused on personal experience.

\section{Impact}

The objective of the training was to impart health knowledge, to encourage changes in lifestyles and to motivate participants to pass on their experiences in their social environments. The effects of the training were investigated with short before and after interviews with all the participants of the first training groups at the three locations $(n=87)$. Detailed structured interviews were conducted with a smaller number of participants $(n=6)$ a year later, in order to find out about longer-term impacts that went beyond the eight-week training stage. In addition, group discussions with participants were conducted in each of the first training groups. In these, the role as health mediator, taken on by the participants, and the activities and the impact on networks and group dynamics were addressed.

In the interviews immediately before and after the training, many participants said that their health-related knowledge was refreshed and that they received good stimulation. A Danish participant expressed what health meant for her before and after the training:

It means the same as always. Only I got something new. And I refreshed my old knowledge. And it gave me a bit of a kick, so that ... whereas before you were slacking, you now want to get going.

Among the most frequently mentioned experiences was gaining basic health knowledge. In Sønderborg, there was a special emphasis on knowledge about nutrition. In Flensburg, participants mainly described the group experience and the information on institutions and services as a benefit.

The participants appreciated receiving information. They found it important, however, to get into action. They described small health-related changes in their daily lives, for example, in their attitudes and thoughts, and also in concrete behaviour, such as dietary changes or exercise. Many participants in Sønderborg set themselves 
targets for diets and weight loss. In Aabenraa, the training consistently focused on health targets. Here, it became apparent that those who were already physically active were able to formulate more concrete targets, and were also able to modify those targets more easily.

A year later, the participants in the structured interviews also reported healthrelated changes, which they mainly saw in small things:

I don't think that any big, ... big changes have happened. But I am still aware of some of the things we learned in the training. So, for example, when I buy groceries, I go for the wholegrain variety and look at the information on the packaging more purposefully ... also what percentage of fat content ... I still do a certain amount of exercise, but it didn't go much ... beyond that, I would say, hmm.

Some participants experienced the small changes that could be implemented in their daily lives as positive; however, others considered them as merely first attempts.

Nearly all the interviewees described personal health targets and their experiences in implementing them. Some said they found it difficult to stick to their targets. They connected this to time restrictions and work obligations. It is possible, though, that they would have needed more support for the step from the motivation phase to implementation, for example, with a concrete action plan or personal coaching (Knoll et al., 2011; Paech et al., 2014).

From the group discussions, it became clear that the participants see their task as health mediators particularly as providing information on professional offerings and referring to appropriate places. Therefore, successful communication and the ability to listen are seen as essential requirements. Some participants see their role as health mediators as being closely bound up with being a role model for a health-promoting lifestyle. The mediators were active, especially in primary networks, such as the family and neighbourhood. They encouraged those in these networks to take part in the training, discussed health-related topics - also, to some extent, controversial ones with family members, or involved them in personal health goals. In secondary networks, the health mediator could, for example, point the way to appropriate professional institutions, or invite work colleagues to public lectures by the health mediators group. It became clear that activities as a health mediator are associated with challenges, such as multiple stresses. One participant said, 'So, I battle more on such fronts as these', and described her attempt to help her husband lose weight and, at the same time, to care for her children and an ill relative. The groups of health mediators also organized joint activities, such as participating in a sporting event. Some health mediators got involved voluntarily in established associations or institutions. Health mediators began, for instance, to build up offerings in an institution for people with mental impairments. We cannot quantify the activities initiated by the mediators because we focused on the content aspects of those small activities. Activities described are very different, for example with regard to their stage of development and their popularity in the community. For this reason, it is very difficult to compare those activities and to express their impact in numbers. The participants in the group discussions also critically reflected on the possibility of being able to measure the activities described and their effects. To some extent, they wanted more feedback and more awareness of their activities from people around them, but they also qualified this with the recognition that more time must be planned for this. In our group discussions, we observed that mediators reported that they were able to acquire more social contacts through the project, and that they intended to maintain them. 
In general, the results showed that the training could encourage participants to address personal targets and take small steps, and that these health targets still played a role in the lives of the health mediators a year later. Thus, the participants had positive experiences that they could pass on in their role as health mediators. Their families played an important role in achieving targets: on the one hand, they provided support and motivation, and, on the other hand, they provided the context for target implementation. Other effects on the participants' social environments and neighbourhoods could only be measured to a limited degree. During the interviews, we learned that towards the end of the project, small activities such as sports or dance events had been organized with the support of neighbourhood associations or round tables (secondary network structures) in which local professional institutions were also involved. This indicates that the project succeeded in stimulating action at different levels of the social network.

\section{Quality of the transnational collaboration}

The collaboration was guided by the five quality dimensions of trust, professionality, transnational added value, personal responsibility and participatory attitudes. The dimensions were selected based on the values and experiences of the project partners, and reflected the advantageous factors of participatory research. At the outset, during and towards the end of the project, moderated discussions took place to reflect on these quality dimensions (see the following discussion). In conclusion, it could be clearly seen that agreement on the quality dimensions was beneficial and created commitment during phases of intensive work, as well as contributing to carrying out the project and the accompanying evaluation on an equal footing. The regular meetings were another helpful factor as they offered space for communication, for the exchange of information on the stages of the project implementation, for the discussion of tasks and for planning the evaluation in the spirit of participatory research. Nevertheless, it became clear that, especially for conceptual tasks such as readjusting the implementation of training, there should have been more time for discussion and exchanging ideas. Much of the meeting time was used for organizational issues.

In the evaluation, challenges were encountered in determining a concrete definition of the transnational value added. The cooperation partners were able, for instance, to agree on defining the jointly developed training concept as being a part of a transnational added value. At the same time, openly dealing with different ways of implementing the training concept and the acceptance of different approaches were also seen as a central aspect of transnational added value. A potential for enhanced use of a transnational added value was clear within the collaboration.

In the sessions used for reflection, it became clear that the collaboration was considered valuable, being composed of a balance between trust, professionalism and personal responsibility; this fact increased the perception of the value of the transnational aspects of the project.

\section{Discussion}

\section{Opportunities and limitations of the evaluation approach}

Altogether, the evaluation approach proved successful. Involving the community practitioners as partners in the evaluation created openness and mutual trust. However, our experience also showed that sufficient time for reflection and exchanging ideas needs to be scheduled, in order to implement the findings more quickly and 
effectively. Methodological obstacles included the transnational and time-consuming implementation, the bilingual collection of qualitative data, and the lack of formal qualifications for carrying out research among the health professionals (to a certain extent). Another challenge was that the design, study and evaluation of the practical approach were interwoven. The project addressed the risk of losing its independent perspective by putting the results up for critical discussion.

\section{Impact of the practical approach}

The approach to offering training for citizens interested in health issues found wide acceptance. The training was very well received, especially in the Danish locations. The results of the evaluation showed that the participants appreciated gaining and updating health knowledge, reflected on healthy lifestyles and managed, to a limited extent, to set individual health targets and implement them with the support of the group and their social environments. However, not all participants achieved this, even when they also perceived the training as generally positive. The results of the interviews are exclusively based on the subjective assessments of the training participants. They do not demonstrate whether a participant actually implemented the health targets or not. Little can be said about the social network effects in the neighbourhood at the time of the evaluation. To this end, it would be necessary to conduct the project over a longer period of time and to evaluate this matter more directly.

\section{Learnings for transnational projects}

We began as a German-Danish project and can pass on the following recommendations for projects that begin transnationally. We developed our concept transnationally, but locally it was adapted to the available professional competencies and the particular environment. In our reflection, we recognized that the acceptance of diversity, such as, for example, in the implementation of the concept, already represents a transnational added value. Significantly more time and personnel resources are needed for more conceptual coordination. In our experience, collaborating transnationally also needs more in the way of interaction - both at the professional level and at the level of the participating citizens. We also recognize that the participating health mediators, who got to know each other at a joint cross-border action day, experienced the exchange as very enriching. In our experience, it is advisable to strongly encourage and promote this exchange between the citizens of the participating communities from the beginning.

\section{Conclusions}

The project Healthy in Everyday Life met with a strong response and was rated positively by the training participants in all three locations. The participants recorded health changes and perceived even small steps to be subjectively important. It was not possible to measure sustainable changes in lifestyle during the project (yet). In order to achieve this, it will be necessary to integrate health targets more systematically in the training, to provide the training facilitators with the relevant tools, and to offer qualified and closer supervision.

The project was successful in stimulating a health-promoting social culture in the neighbourhoods and municipalities. During the course of the project, small local activities and offers were initiated that have the potential to generate long-term effects in the neighbourhoods and municipalities. However, concrete developments cannot be anticipated yet. In order to continue the project and measure the effects of the 
network-promoting approach that take longer to become apparent in neighbourhoods, more time would be required.

The implementation of the transnational collaboration in participatory research was complex; however, the project proved to be innovative and productive. The participants could inspire and learn from each other when it came to developing and implementing local health-promotion activities. This form of collaboration requires a lot of time and sharing of ideas. Resources are also necessary to prepare the health professionals for the research tasks and to offer them opportunities to become involved. We noticed particularly how important it is to connect the analysis of the evaluation findings to further development in practice. For this, the project duration was too short.

The use of sessions for reflection on the project, which were integrated into the evaluation, were a new element for participatory research. Due to restricted financial resources, it was conducted by means of self-evaluation. For research projects such as ours, we recommend building in reflection loops and the use of external services, such as team supervision and team development, for additional support. Our experience has shown that reflection allows a good culture of collaboration to develop; this in turn, has a positive impact on project success.

\section{Acknowledgements}

We would like to thank Anette Krogh, Søren Schmidt Josefsen, Anette Anker, Camilla Galthen, Stephan Rehberg, Michael Kraus, Martin Oldenburg, Jana Dördelmann and Eva Weidner for their collaboration. Special thanks are due to the health mediators who participated in the evaluation with great interest and commitment.

\section{Funding}

The work was funded by the European Commission (funding programme Interreg 4A Syddanmark-Schleswig-K.E.R.N., reference number CCI-2007CB163PO056, grant or project number 112-2.1-12).

\section{Notes on the contributors}

Petra Wihofszky is Professor of Health Sciences, with a focus on nursing science, at the Esslingen University of Applied Sciences, in Germany. Previously, she was Assistant Professor in the Department of Health Psychology and Health Education at the European University of Flensburg located in the German-Danish border region, teaching and researching community health promotion and participation.

Annika Sternberg is currently working as a research assistant in the Rehabilitation and Work Section of the Institute of Social Medicine and Epidemiology at the University of Lübeck, Germany, with a focus on vocational rehabilitation strategies. Previously, she worked on community health promotion in the Department of Health Psychology and Health Education at the European University of Flensburg.

\section{References}

Bär, G. (2012) 'Partizipation im Quartier - Gesundheitsförderung mit vielen Adressaten und Dynamiken'. In Rosenbrock, R. and Hartung, S. (eds) Handbuch Partizipation und Gesundheit. Bern: Huber, 172-82. 
Bär, G. (2013) 'Wissenschaftliche Begleitung, formative Evaluation und partizipative Forschung: Methodische Fußangeln für Wissenschafts-Praxis-Partnerschaften'. Prävention und Gesundheitsförderung, 8 (3), 155-62.

Bergold, J. and Thomas, S. (2012) 'Partizipative Forschungsmethoden: Ein methodischer Ansatz in Bewegung'. Forum: Qualitative Social Research, 13 (1), Article 30. Online. https://tinyurl.com/ c44hse8 (accessed 31 May 2019).

Borg, M., Karlsson, B., Kim, H.S. and McCormack, B. (2012) 'Opening up for many voices in knowledge construction'. Forum: Qualitative Social Research, 13 (1), Article 1. Online. https://tinyurl.com/y2mtfbj7 (accessed 31 May 2019).

Brandes, S. and Schaefer, I. (2013) 'Partizipative Evaluation in Praxisprojekten: Chancen und Herausforderungen'. Prävention und Gesundheitsförderung, 8 (3), 132-7.

Brown, A. (2013) Action and Research: Community-based research at the Wellesley Institute. Toronto: Wellesley Institute. Online. https://tinyurl.com/yxjgl79y (accessed 31 May 2019).

Bruns, W. (2013) Gesundheitsförderung durch soziale Netzwerke: Möglichkeiten und Restriktionen. Wiesbaden: Springer.

Faltermaier, T. (2008) 'Health psychology and health promotion: Salutogenetic and competenceoriented approaches in an educational context'. Zeitschrift für Gesundheitspsychologie, 16 (3), 123-6.

Flicker, S., Savan, B., McGrath, M., Kolenda, B. and Mildenberger, M. (2008) '“If you could change one thing...": What community-based researchers wish they could have done differently'. Community Development Journal, 43 (2), 239-53.

Hartung, F.-M. and Renner, B. (2014) 'The need to belong and the relationship between loneliness and health'. Zeitschrift für Gesundheitspsychologie, 22 (4), 194-201.

Hicks, S., Duran, B., Wallerstein, N., Avila, M., Belone, L., Lucero, J., Magarati, M., Mainer, E., Martin, D., Muhammad, M., Oetzel, J., Pearson, C., Sahota, P., Simonds, V., Sussman, A., Tafoya, G. and White Hat, E. (2012) 'Evaluating community-based participatory research to improve communitypartnered science and community health'. Progress in Community Health Partnerships: Research, Education, and Action, 6 (3), 289-99.

Jordan, S. and Von der Lippe, E. (2012) 'Angebote der Prävention - Wer nimmt teil?'. GBE Kompakt, 3 (5), 1-9. Online. https://tinyurl.com/y5w65dln (accessed 31 May 2019).

Kienle, R., Knoll, N. and Renneberg, B. (2006) 'Soziale Ressourcen und Gesundheit: Soziale Unterstützung und dyadisches Bewältigen'. In Renneberg, B. and Hammelstein, P. (eds) Gesundheitspsychologie. Heidelberg: Springer, 107-22.

Knoll, N. and Burkert, S. (2009) 'Soziale Unterstützung und soziale Netzwerke als Ressource der Krankheitsbewältigung'. In Schaeffer, D. (ed.) Bewältigung chronischer Krankheit im Lebenslauf. Bern: Huber, 223-43.

Knoll, N., Scholz, U. and Rieckmann, N. (2011) Einführung in die Gesundheitspsychologie. 2nd ed. München: Reinhardt.

Kochinka, A. (2010) 'Beobachtung'. In Mey, G. and Mruck, K. (eds) Handbuch Qualitative Forschung in der Psychologie. Wiesbaden: VS Verlag für Sozialwissenschaften, 449-61.

Kuckartz, U. (2014) Qualitative Inhaltsanalyse: Methoden, Praxis, Computerunterstützung. 2nd ed. Weinheim: Beltz Juventa.

Lantz, P.M., Viruell-Fuentes, E., Israel, B.A., Softley, D. and Guzman, R. (2001) 'Can communities and academia work together on public health research? Evaluation results from a community-based participatory research partnership in Detroit'. Journal of Urban Health, 78 (3), 495-507.

Larsen, E.L. and Stock, C. (2011) 'Capturing contrasted realities: Integrating multiple perspectives of Danish community life in health promotion'. Health Promotion International, 26 (1), 14-22.

Lenz, A. (2000) 'Förderung sozialer Ressourcen - eine gemeindepsychologische Perspektive'. Gruppe, Interaktion, Organisation: Zeitschrift für Angewandte Organisationspsychologie (GIO), 31 (3), 277-302.

Mohnen, S.M., Groenewegen, P.P., Völker, B. and Flap, H. (2011) ‘Neighborhood social capital and individual health'. Social Science and Medicine, 72 (5), 660-7.

Nestmann, F. (2009) 'Netzwerkintervention und soziale Unterstützungsförderung'. In Lenz, K. and Nestmann, F. (eds) Handbuch Persönliche Beziehungen. Weinheim: Juventa, 955-77.

Paech, J., Fleig, L., Pomp, S. and Lippke, S. (2014) 'Soziale Unterstützung als Wegbereiter für Planungsprozesse: Körperliche Aktivität nach der medizinischen Rehabilitation'. Zeitschrift für Gesundheitspsychologie, 22 (2), 39-49.

Richter, M. and Hurrelmann, K. (2011) 'Determinanten von Gesundheit'. In Bundeszentrale für gesundheitliche Aufklärung (BZgA) Leitbegriffe der Gesundheitsförderung und Prävention: Glossar zu Konzepten, Strategien und Methoden. Gamburg: Verlag für Gesundheitsförderung, 45-8. 
Schwarzer, R. (2008) 'Modeling health behavior change: How to predict and modify the adoption and maintenance of health behaviors'. Applied Psychology, 57 (1), 1-29.

Stolzenberg, R., Berg, G. and Maschewsky-Schneider, U. (2012) 'Healthy upbringing of children through the empowerment of women in a disadvantaged neighbourhood: Evaluation of a peer group project'. Journal of Public Health, 20 (2), 181-92.

Trojan, A. and Süß, W. (2011) 'Soziale Netzwerke und Netzwerkförderung'. In Bundeszentrale für gesundheitliche Aufklärung (BZgA) Leitbegriffe der Gesundheitsförderung und Prävention: Glossar zu Konzepten, Strategien und Methoden. Gamburg: Verlag für Gesundheitsförderung, 501-3.

Weyers, S. (2008) 'Soziale Ungleichheit, soziale Beziehungen und Gesundheitsverhalten'. In Bauer, U., Bittlingmayer, U.H. and Richter, M. (eds) Health Inequalities: Determinanten und Mechanismen gesundheitlicher Ungleichheit. Wiesbaden: VS Verlag für Sozialwissenschaften, 257-70.

Weyers, S., Dragano, N., Möbus, S., Beck, E.-M., Stang, A., Möhlenkamp, S., Jöckel, K.H., Erbel, R. and Siegrist, J. (2010) 'Poor social relations and adverse health behaviour: Stronger associations in low socioeconomic groups?'. International Journal of Public Health, 55 (1), 17-23.

Whitehead, M. and Dahlgren, G. (1991) 'What can be done about inequalities in health?'. The Lancet, 338 (8774), 1059-63.

Wihofszky, P. (2013) 'Die Praxis der Gesundheitsförderung zwischen Top-down und Bottom-up: Ansätze partizipativen Planens und Forschens in der Konzeptentwicklung'. Prävention und Gesundheitsförderung, 8 (3), 181-90.

Wright, M.T. (2013) 'Was ist Partizipative Gesundheitsforschung? Positionspapier der International Collaboration for Participatory Health Research'. Prävention und Gesundheitsförderung, 8 (3), 122-31.

Wright, M.T., Springett, J. and Kongats, K. (2018) 'What is participatory health research?'. In Wright, M.T. and Kongats, K. (eds) Participatory Health Research: Voices from around the world. Cham: Springer, 3-15. 